\title{
Dynamic robust stabilization of stochastic differential control systems
}

\begin{abstract}
The stabilization of stochastic differential control systems by means of dynamic feedback laws is provided. We extend the well-known Artstein-Sontag theorem to derive the necessary and sufficient conditions for the dynamic robust stabilization of stochastic differential systems. An explicit formula for feedback law exhibiting dynamic robust stability in probability is established, and a numerical example is also given to illustrate our results.
\end{abstract}

Keyword: Dynamic control Lyapunov function; Robust stability in probability; Stochastic differential control system. 